$06,10,11$

\title{
Антиферродисторсионная мягкая мода в кристалле $\mathrm{PbZr}_{0.024} \mathrm{Ti}_{0.976} \mathrm{O}_{3}$
}

\author{
(C) С.Б. Вахрушев ${ }^{1}$, Ю.А. Бронвальд ${ }^{1,2}$, К.А. Петрухно ${ }^{1,2}$, C.А. Удовенко ${ }^{1,2}$, \\ И.Н. Леонтьев ${ }^{3}$, А. Bosak ${ }^{4}$ \\ ${ }^{1}$ Физико-технический институт им. А.Ф. Иофрфе РАН, \\ Санкт-Петербург, Россия \\ ${ }^{2}$ Санкт-Петербургский политехнический университет Петра Великого, \\ Санкт-Петербург, Россия \\ ${ }^{3}$ Южный фредеральный университет, \\ Ростов-на-Дону, Россия \\ ${ }^{4}$ Swiss-Norwegian Beamlines at ESRF, \\ Grenoble, France \\ E-mail: k.a.petroukhno@gmail.com \\ Поступила в Редакцию 13 мая 2021 г. \\ В окончательной редакции 13 мая 2021 г. \\ Принята к публикации 13 мая 2021 г.
}

\begin{abstract}
Используя метод неупругого рассеяния синхротронного излучения обнаружена антиферродисторсионная мягкая мода в точке $\mathrm{M}$ зоны Бриллюэна в кристалле $\mathrm{PbZr}_{0.024} \mathrm{Ti}_{0.976} \mathrm{O}_{3}$. Проведенный теоретико-групповой анализ и расчеты неупругих структурных факторов позволили однозначно соотнести обнаруженное критическое возбуждение с разворотами кислородных октаэдров. Прослежена температурная эволюция частоты мягкой моды и показано, что она следует закону Кюри-Вейсса с температурой Кюри $T_{\mathrm{AFD}}=438 \pm 5 \mathrm{~K}$, близкой к сегнетоэлектрической температуре Кюри $T_{\mathrm{FE}}=479.5 \pm 5 \mathrm{~K}$. Частота антиферродисторсионной мягкой моды оказалась меньше частоты дважды вырожденного акустического колебания в М-точке, что делает неизбежным возникновение межмодового взаимодействия и антикроссинга при отклонении от границы зоны Бриллюэна.
\end{abstract}

Ключевые слова: сегнетоэлектрики; антисегнетоэлектрики; фазовые переходы; динамика решетки; неупругое рассеяние СИ.

DOI: 10.21883/FTT.2021.10.51405.113

\section{1. Введение}

В последние годы наблюдается рост интереса к антисегнетоэлектрическим (АСЭ) кристаллам. Этот рост связан как с возможностями их практического применения как основы для создания быстрых накопителей электрической энергии и электрокалорических охлаждающих устройств, так и с интересной и богатой физикой этих соединений. По-видимому, наиболее изученными АСЭ-материалами являются цирконат свинца $\mathrm{PbZrO}_{3}$ (PZO) и твердые растворы на его основе $\mathrm{PbZr}_{1-x} \mathrm{Ti}_{x} \mathrm{O}_{3}$ (PZT) с $x \leq 0.06$ и изоморфные им соединения, например, $\mathrm{PbHfO}_{3}$. В параэлектрической фазе все эти соединения имеют структуру кубического перовскита. Следует отметить, что в большинстве этих соединений фазовый переход в АСЭ-фазу происходит через промежуточную сегнетоэлектрическую (СЭ) фазу $[1,2]$. В монокристаллах чистого PZO CЭ-фаза существует в очень узком температурном интервале $[3,4]$, в то время как в керамиках цирконата свинца промежуточная СЭ-фаза наблюдается в довольно широкой температурной области [5]. В зависимости от концентрации компонентов в твердых растворах и от внешних условий в соединениях группы PZO наблюдается большое разнообразие структурных состояний, включая упомянутые АCЭ и СЭ фазы [6] фазы, а также несоразмерные структуры $[7,8]$. Структура АСЭ-фаз хорошо изучена; обнаружено сосуществование двух параметров порядка - собственно антисегнетоэлектрического, характеризуемого волновым вектором $q_{\Sigma}=(1 / 41 / 40)$ и антиферродисторсионного, характеризуемого волновым вектором $q_{R}=\left(\begin{array}{lll}1 / 2 & 1 / 2 & 1 / 2\end{array}\right)$.

В работе [8] приведены результаты расшифровки несоразмерной структуры $\mathrm{PbHfO}_{3}$. В тоже время однозначное решение структуры СЭ-фазы PZT с $x \leq 0.06$ по прежнему отсутствует несмотря на большое число работ посвященных этой проблеме [9-12]. Сложность расшифровки структуры этих соединений связана с сосуществованием параметров порядка различной симметрии. Если полагать, что переход из параэлектрической (ПЭ) в СЭ-фазу является собственным сегнетоэлектрическим - то первичным параметром порядка следует считать поляризацию. Однако в СЭ-фазе возникают сверхструктурные отражения в М-точке зоны Бриллюэна (ЗБ) и дополнительные сателлитные отражения $[13,14]$. Возникающая М-сверхструктура не может быть соотнесена с антиферродисторсионным (АФД) параметром порядка (ПП), связанным с параллельными разворотами кислородных октаэдров в соседних плоскостях, поскольку отсутствует специфический для такого ПП закон погасания [10]. Более того, как было показано в работе [14] возникающий сложный набор сателлитных отражений не укладывается 
в предлагавшуюся в статьях $[10,15]$ картину антифазных доменов. Одним из наиболее эффективных путей поиска ответа на вопрос о формировании структуры СЭ-фазы является прослеживание релевантной критической динамики.

Вопрос о динамике решетки материалов PZO рассматривался многократно. Было прослежено поведение СЭ мягкой моды в центре ЗБ [16]. Были предложены динамические модели определяющие возникновение АСЭ-параметра порядка $[17,18]$ и выявлены возбуждения ответственные за несоразмерный ФП. Большое внимание было уделено вопросу мягкой моды в $\mathrm{R}$-точке. B ряде теоретических работ именно смягчение АФД-моды рассматривалось как основное, инициирующее АСЭ ФП. Для чистого PZO эта концепция не была подтверждена, и было показано, что R-ПП возникает по триггерному механизму $[17,18]$. В то же время в работе [19] была выявлена конденсация $\mathrm{R}$-моды в кристалле $\mathrm{PbHfO}_{3}$ и было предположено, что именно она является, „первичной“. В отличие от $\mathrm{R}$-точки динамика решетки в окрестности М-точки остается мало изученной. Оптические методы не позволяют непосредственно исследовать возбуждения на границе зоны Бриллюэна. Оптимальным методом для изучения кислородных АФД-мод является неупругое рассеяние нейтронов, однако монокристаллы PZT слишком малы. На практике единственным способом исследования фононной динамики в них оказывается неупругое рассеяние рентгеновского (синхротронного) излучения. Косвенная информация о критической динамике может быть получена из анализа температурной эволюции диффузного рассеяния (ДР). Интенсивное диффузное рассеяние в окрестности М-точки как в чистом PZO, так и твердых растворах РZT, было описано в ряде работ [20-22]. Однако, в большинстве случаев измерения проводились при фиксированной температуре. При этом в случае рассеяния СИ наблюдалось существенно асимметричное распределение рассеяния, а в единственной работе, в которой было изучено диффузное рассеяние нейтронов [21] максимум интенсивности приходился на М-точку, однако никакой информации о температурной зависимости этого максимума приведено не было. Принципиальное значение для интерпретации наблюдаемой картины ДР имеют законы погасания. Сильное ДР наблюдается в точках обратного пространства $(h+1 / 2 k+1 / 2 l)$ при $h \neq k$. Это рассеяние практически исчезает при $h=k$. Такие правила отбора характерны для кислородной АФД-моды. В работе [22] рассмотрена температурная эволюция ДР в районе М-точки в $\mathrm{PbZr}_{0.993} \mathrm{Ti}_{0.007} \mathrm{O}_{3}$.

В статье Глинки и др. [23] приведены результаты исследования динамики решетки кристалла $\mathrm{PbZr}_{0.475} \mathrm{Ti}_{0.525} \mathrm{O}_{3}$. Особое внимание было уделено изучению фононных резонансов в М-точке. При анализе данных кислородная АФД-мода была исключена из рассмотрения, по-видимому, исходя из предположения, что ее вклад крайне мал вследствие относительной малости атомных факторов рассеяния кислорода, по сравнению с атомными факторами рассеяния свинца. Необходимо, однако, отметить, что этот аргумент, однозначно важный в случае брэгговского рассеяния становится спорным в случае однофононного неупругого рассеяния. Вопрос о принципиальной наблюдаемости мягкой кислородной АФД-моды в экспериментах по неупругому рассеянию рентгеновского излучения будет рассмотрен ниже.

Суммируя вышесказанное, необходимо отметить, что на сегодняшний день отсутствуют экспериментальные данные, подтверждающие или опровергающие существование АФД мягкой моды в М-точке в кристаллах. Следует отметить, что в теоретических работах, посвященных динамике решетки PZO и твердых растворах на его основе поведению кислородной моды в М-точке уделено крайне мало внимания. В статье [24] показано, что в кубической фазе $\mathrm{PZO}$ при $0 \mathrm{~K}$ частота $M_{3}$ моды является мнимой и приблизительно равной частоте $R_{25}$ моды. Результаты экспериментального исследования температурной эволюции неупругого рассеяния СИ в $\mathrm{PbZr}_{0.993} \mathrm{Ti}_{0.007} \mathrm{O}_{3}$ при волновых векторах вдоль $\Sigma$-линии $(q q 0)$, включая М-точку представлены в работе [25]. Был выявлен рост интенсивности рассеяния в области малых переданных энергий при приближении к М-точке, однако подробный анализ формы линии и вопросов смягчения мод не проводился.

Для получения ответа на вопрос о частоте кислородной АФД-моды и ее температурной эволюции нами было изучено неупругое рассеяние СИ в монокристалле $\mathrm{PbZr}_{0.024} \mathrm{Ti}_{0.976} \mathrm{O}_{3}$ (PZT2.4) В этом кристалле наблюдаются 2 последовательных фазовых перехода: (1) из параэлектрической в сегнетоэлектрическую при $T_{1} \approx 520 \mathrm{~K}$, сопровождающийся возникновением М-сверхструктуры и из СЭ- в АСЭ-фазу при $T_{2} \approx 420 \mathrm{~K}$. Измерения были выполнены в параэлектрической фазе в температурном интервале от $550 \mathrm{~K}$ до $700 \mathrm{~K}$.

\section{2. Методика и предварительный анализ}

Монокристалл PZT2.4 был выращен в Южном федеральном университете по методике, описанной в [26]. Для исследования неупругого рассеяния были изготовлены стержнеобразные образцы длиной порядка $1 \mathrm{~mm}$ прямоугольного $\left(\sim 50 \times 150 \mu \mathrm{m}^{2}\right)$ сечения. Образец вырезался из монокристалла большего размера, шлифовался и протравливался в кипящей соляной кислоте. Измерения проводились на спектрометре, установленном на линии ID28 Европейского синхротронного источника (ESRF). Большая часть измерений проводилась при энергии излучения $21.747 \mathrm{keV}$ (монохроматор $\mathrm{Si}(11,11,11)$, разрешение по энергии $\approx 1.7 \mathrm{meV})$. Нагрев осуществлялся со струей азота, с использованием Cryostream 700 Plus. Точность поддержания температуры составляла $\approx 1 \mathrm{~K}$. Все измерения проводились в плоскости обратной решетки $(h k 0)$. 


\section{3. Наблюдаемость кислородной мягкой моды в кристаллах типа PZT}

Как правило, считается, что надежно выделить вклад в рассеяние рентгеновского излучения от легких элементов в присутствии тяжелых оказывается затруднительным. Действительно, когда мы говорим о Брэгговском рассеянии, то парциальный вклад входящих в состав вещества элементов в суммарную интенсивность рассеяния пропорционален величине атомного фактора рассеяния $f$, т. е. в первом приближении, атомному номеру $Z$. В случае неупругого рассеяния зависимость от $f$, в значительной степени сохраняется, но возникает дополнительная зависимость от амплитуды атомных смещений. Функция рассеяния для однофононного неупругого рассеяния рентгеновского излучения $S_{J}(\mathbf{Q}, \omega)$ может быть записана в виде:

$$
S_{l}(\mathbf{Q}, \omega)=\left[F_{j}(\mathbf{Q}, \mathbf{q})\right]^{2} G_{j}\left(\omega, \omega_{j}(\mathbf{q}, T)\right) .
$$

Здесь $j$ - номер фононной моды, $\mathbf{Q}-$ переданный волновой вектор, $\hbar \omega-$ переданная энергия, $\mathbf{q}-$ приведенный волновой вектор, $T-$ температура, $\omega_{j}(\mathbf{q})-$ частота фонона с волновым вектором q, принадлежащего моде $j$,

$$
\begin{aligned}
F_{j}(\mathbf{Q}, \mathbf{q}) & =\Sigma_{l} \frac{f_{l}}{\sqrt{M_{l}}} \mathbf{Q \mathbf { e } _ { l } ^ { j }}(\mathbf{q}) \exp \left(i \mathbf{Q \mathbf { Q } _ { l }}\right) \exp \left(-W_{l}\right) \\
& =\Sigma_{l} b_{l} \mathbf{Q} \mathbf{e}_{l}^{j}(\mathbf{q}) \exp \left(i \mathbf{Q} \mathbf{r}_{l}\right) \exp \left(-W_{l}\right)
\end{aligned}
$$

- неупругий структурный фактор, $l$ нумерует атомы в элементарной ячейке, $\mathbf{e}-$ собственный вектор смещения, $\mathbf{r}-$ положение атома в элементарной ячейке, $f_{l}-$ атомный фактор рассеяния и $M_{l}$ масса атома $l$. Легко видеть, что можно ввести эффективную амплитуду рассеяния $b_{l}=f_{l} / \sqrt{M_{l}}$. Динамический отклик $G_{j}\left(\omega, \omega_{j}(\mathbf{q}, T)\right)$ описывается выражением

$$
G_{j}\left(\omega, \omega_{j}(\mathbf{q}, T)\right)=\left[n(\omega)+\frac{1}{2} \pm \frac{1}{2}\right] \operatorname{Im}\left(\chi_{j}(\mathbf{q})\right),
$$

где $n(\omega)$ - фактор заселенности, знак „+““ соответствует процессам с рождением фонона, а „-“ - c его поглощением, $\operatorname{Im}\left(\chi_{j}(\mathbf{q})\right)$ мнимая часть обобщенной восприимчивости, в простейшем случае описываемая затухающим гармоническим осциллятором

$$
\chi_{j}=1 /\left(\omega^{2}-\omega_{j}^{2}+i \Gamma_{j} \omega\right),
$$

где $\Gamma_{j}-$ константа затухания. В высокотемпературном приближении $k T \ll \hbar \omega$, как правило корректном для случая мягкой моды, интегральный по энергии динамический отклик оказывается обратно пропорциональным квадрату частоты фонона

$$
\int_{-\infty}^{\infty} G_{j}\left(\omega, \omega_{j}(\mathbf{q}, T)\right) \propto 1 / \omega_{j}^{2} .
$$

Основываясь на приведенных выражениях, можно оценить наблюдаемость кислородной АФД мягкой моды в соединении типа PZO. Следуя [23] будем считать что основной вклад в дважды вырожденную $M_{5^{\prime}}$-моду дают смещения ионов свинца. АФД $M_{3}$-мода является чисто кислородной. Как отмечено выше, полагаем, что атомные факторы рассеяния пропорциональны атомному номеру. Тогда получаем

$$
\frac{b_{\mathrm{O}}^{2}}{b_{\mathrm{Pb}}^{2}} \approx\left(\frac{z_{\mathrm{O}}^{2}}{M_{\mathrm{O}}}\right) \frac{z_{\mathrm{Pb}}^{2}}{M_{\mathrm{Pb}}} \approx 0.11 .
$$

Таким образом, можно ожидать, что неупругий структурный фактор АФД-моды не является пренебрежимо малым (точное значение зависит от переданного волнового вектора $\mathbf{Q}$ ). Фактором, определяющим наблюдаемость кислородной моды, оказывается величина динамического отклика. В общем случае частоты колебаний, связанных со смещением тяжелых атомов оказываются более низкими, чем в случае легких атомов. Однако особенностью мягких мод является резкое понижение их частоты, по мере приближения к критической температуре. Так, в сегнетоэлектрике релаксоре $\mathrm{Na}_{0.5} \mathrm{Bi}_{0.5} \mathrm{TiO}_{3}$, в котором также наблюдается сосуществование сегнетоэлектрической и антиферродисторсионных мод, энергия $M_{3}$-моды вблизи температуры перехода из кубической в тетрагональную фазу опускается почти до $0.5 \mathrm{meV}$ [27].

\section{4. Теоретико-групповой анализ и правила отбора для неупругого рассеяния}

Для дальнейшего рассмотрения запишем координаты входящих в структуру атомов.

$\mathrm{Pb}:\left(\begin{array}{lll}0 & 0 & 0\end{array}\right) ; \operatorname{Zr}(\mathrm{Ti}):\left(\begin{array}{lllll}1 / 2 & 1 / 2 & 1 / 2\end{array}\right) ; \quad \mathrm{O}_{1}:\left(\begin{array}{lll}1 / 2 & 1 / 2 & 0\end{array}\right)$; $\mathrm{O}_{2}:\left(\begin{array}{lll}1 / 2 & 0 & 1 / 2\end{array}\right) ; \mathrm{O}_{3}:\left(\begin{array}{lll}0 & 1 / 2 & 1 / 2\end{array}\right)$.

Разложение механического представления на неприводимые в М-точке зоны Бриллюэна для различных ионов $D_{j}$, где $j-$ тип иона, имеет вид (для неприводимых представлений используем обозначения из работы [28]):

$$
\begin{gathered}
D_{\mathrm{Pb}}=M_{2^{\prime}}+M_{5^{\prime}} ; \quad D_{\mathrm{Zr}(\mathrm{Ti})}=M_{3^{\prime}}+M_{5^{\prime}} ; \\
D_{\mathrm{O}}=M_{1}+M_{2}+M_{2^{\prime}}+M_{3}+M_{4}+M_{5}+M_{5^{\prime}} .
\end{gathered}
$$

Неприводимые представления являются $M_{1}, M_{2}, M_{2^{\prime}}$, $M_{3}, M_{3^{\prime}}, M_{4}$ одномерными и соответствующие им фононные моды (которые, как и во введении мы будем называть по их неприводимым представлениям) не вырождены, а двумерные представления $M_{5}$ и $M_{5}$, описывают дважды вырожденные моды колебаний. Собственные вектора колебаний $M_{2^{\prime}}, M_{3^{\prime}}$ и $M_{5}$ направлены вдоль оси $Z$ и в плоскости $(h k 0)$ наблюдаться не могут, вследствие зануления скалярного произведения в формуле 2. Чисто кислородные моды $M_{2}$, и $M_{3}$ погасают в точках $(h+1 / 2, k+1 / 2, l)$, при условии $h=k$ [29], при этом мода $M_{3}$, как обсуждалось выше, связана с 

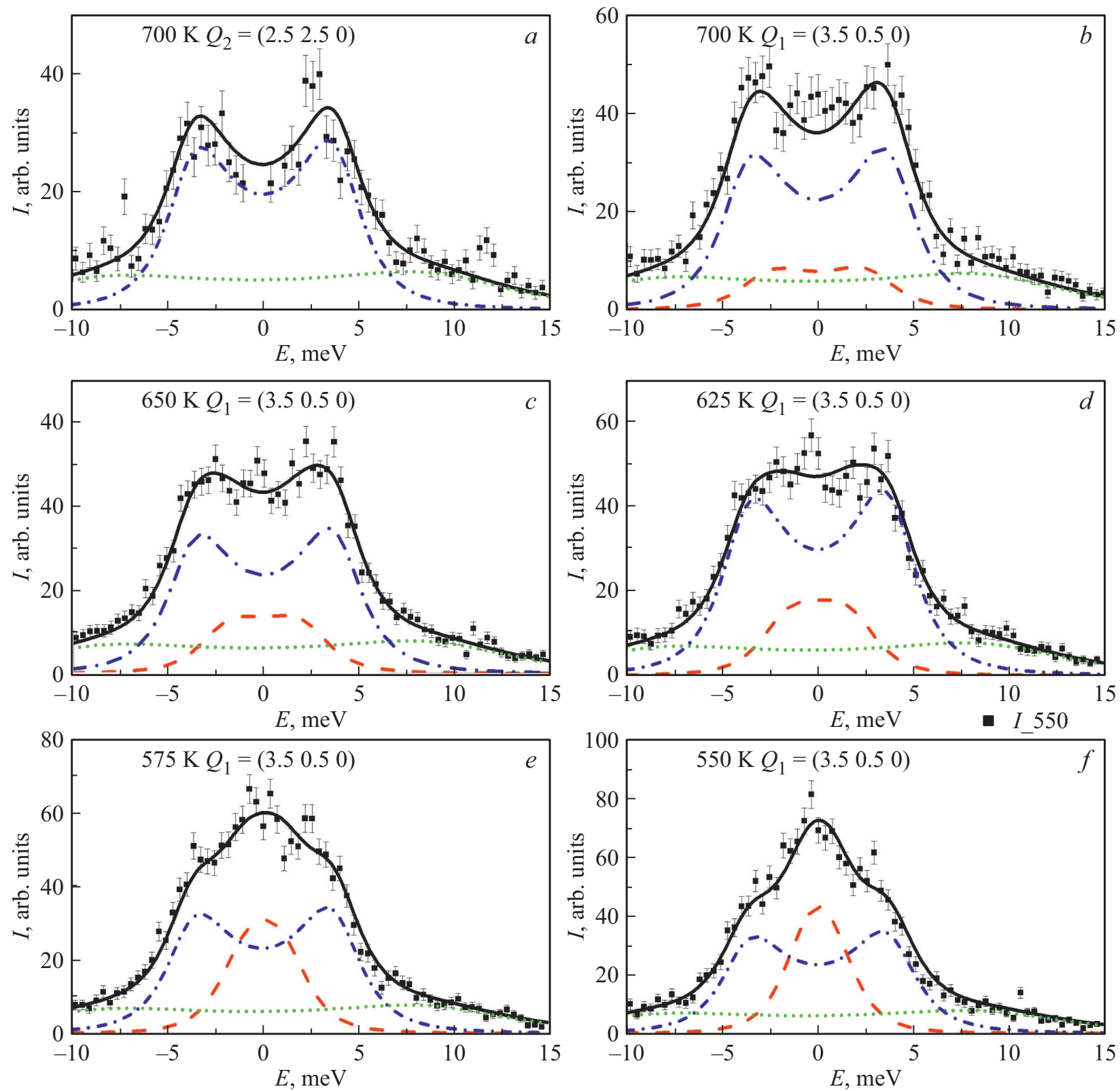

Рис. 1. Температурная эволюция формы спектров неупругого рассеяния СИ в М-точке зоны Бриллюэна. $(a)-Q_{2}=(2.52 .50)$ $700 \mathrm{~K}$ (3 точки в центре выброшены); $(b),(c),(d),(e)$, и $(f) Q_{1}=(3.50 .50)$. Суммарная рассчитанная интенсивность показана сплошной линией, вклады акустической, АФД и оптической мод - штрихпунктирной, штриховой и пунктирной, соответственно.

вращением кислородного октаэдра как целого и может иметь очень низкую частоту, в то время как мода $M_{2}$ связана с деформацией октаэдра и, как правило, имеет высокую частоту [24] и вероятность ее наблюдения в эксперименте по неупругому рассеянию рентгеновского излучения крайне мала. Для мод $M_{1}, M_{4}$ правила отбора отсутствуют.

\section{5. Результаты и обсуждение}

На рис. 1 приведены фононные спектры, измеренные в точке $Q_{1}=\left(\begin{array}{lll}3.5 & 0.5 & 0\end{array}\right)$ при $T=700 \mathrm{~K}, 650 \mathrm{~K}, 575 \mathrm{~K}$ и
$550 \mathrm{~K}$ и в точке $Q_{2}=\left(\begin{array}{lll}2.5 & 2.50\end{array}\right)$ при $700 \mathrm{~K}$. Спектры в точке $Q_{2}$, аналогичны описанным в работе [23], для морфотропного PZT и согласуются с данными [22] для $\mathrm{PbZr}_{0.993} \mathrm{Ti}_{0.007} \mathrm{O}_{3}$. Низкочастотная компонента, с максимумом в районе $4 \mathrm{meV}$, очевидно, связана с дважды вырожденной акустической модой $M_{5^{\prime}}^{A}$ (слияние поперечной и продольной акустических дисперсионных кривых на границе зоны), а высокочастотная, проявляющаяся в районе $10 \mathrm{meV}$, обусловлена дважды вырожденной оптической модой также имеющей симметрию $M_{5^{\prime}}^{O}$. Спектр в точке $Q_{1}$ при $700 \mathrm{~K}$ мало отличается от наблюдаемого в точке $Q_{2}$, однако при понижении температуры и приближении к фазовому переходу в 
СЭ фазу четко проявляется дополнительная низкоэнергетическая компонента. Этот низкочастотный вклад не может быть обусловлен модой $M_{2^{\prime}}$, поскольку она не может наблюдаться в $(h k 0)$ плоскости, как отмечалось выше. Сравнение с теоретическими расчетами позволяет соотнести низкочастотное рассеяние в точке $Q_{1}$ с АФД модой $M_{3}$.

Экспериментально наблюдаемые в $Q_{1}$ спектры являются плохо разрешенными, что дает большой произвол при аппроксимации их суммой независимых фононных резонансов (3). Чтобы существенно повысить однозначность получаемых результатов мы использовали два приближения. Мы полагали, что частоты акустического $M_{5^{\prime}}^{A}$ и оптического $M_{5^{\prime}}^{O}$ колебаний не зависят от температуры. Для колебания $M_{5^{\prime}}^{A}$ это следует из результатов, полученных для чистого цирконата свинца [17] и твердого раствора $\mathrm{PbZr}_{0.985} \mathrm{Ti}_{0.015} \mathrm{O}_{3}[22]$.

Мы также считали, что $M_{5}^{A}$ практически полностью связана со смещениями иона свинца. Это предположение поддерживается, в первую очередь, результатами расчетов чистого $\mathrm{PbZrO}_{3}$ методами молекулярной динамики [20].

Измеренные спектры описывались как сумма четырех фононных резонансов

$$
\begin{aligned}
I= & A(T)\left[\left[F_{A 1}^{2}(\mathbf{Q}) G_{A}+F_{A 2}^{2}(Q) G_{A}\right]\right. \\
& \left.+F_{\mathrm{AFD}}^{2}(\mathbf{Q}) G_{\mathrm{AFD}}(T)+F_{\mathrm{O}}^{2}(\mathbf{Q}) G_{\mathrm{O}}\right],
\end{aligned}
$$

где $A(T)$ - температурно-зависимый шкальный множитель, $G_{A}, G_{\mathrm{AFD}}$ и $G_{\mathrm{O}}$ - динамические отклики а $F_{A 1}^{2}, F_{A 2}^{2}, F_{\mathrm{AFD}}^{2}$ и $F_{\mathrm{O}}^{2}$ неупругие структурные факторы для дважды вырожденной акустической, антиферродисторсионной и оптической мод. Неупругие структурные факторы для акустической и АФД мод рассчитывались по формуле (2). Собственный вектор АФД-моды полностью определяется одномерным неприводимым представлением $M_{3}$, однократно входящим в разложение механического представления и имеет вид (представлен как прямая сумма двух векторов, относящихся к $\mathrm{O}_{2}$ и $\mathrm{O}_{3}$, соответственно): $e_{\mathrm{AFD}}=e_{\mathrm{AFD}}^{\mathrm{O}_{1}} \oplus e_{\mathrm{AFD}}^{\mathrm{O}_{2}}=(100) \oplus(0-10)$. Акустическая мода дважды вырождена и может быть представлена как сумма двух мод. В качестве собственных векторов (с учетом сделанного предположения о доминирующей роли смещений свинца) может быть выбрана любая ортонормированная пара векторов в плоскости $X-Y$. В принципе всегда можно выбрать эту пару таким образом, что ненулевое значение будет иметь неупругий структурный фактор только для одной моды, однако в этом случае пришлось бы разворачивать базис для каждого переданного волнового вектора. Нами был выбран вариант

$$
e_{A}^{1}=(1 / \sqrt{2},-1 / \sqrt{2}, 0), \quad e_{A}^{2}=(1 / \sqrt{2}, 1 / \sqrt{2}, 0) .
$$

Такой выбор позволяет в дальнейшем использовать написанный нами скрипт для обработки результатов в

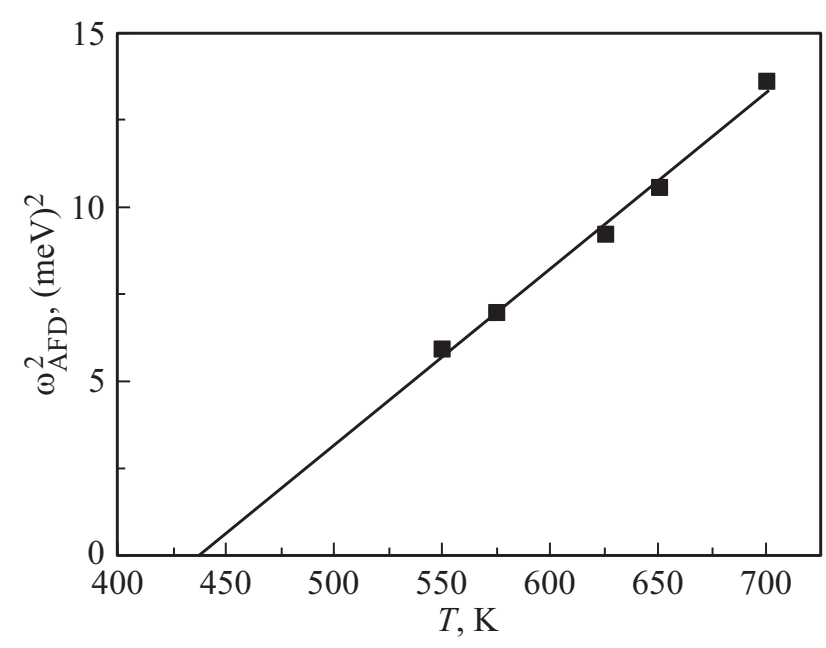

Рис. 2. Температурная зависимость квадрата частоты антиферродисторсионной моды.

произвольных точках $\Sigma$-линии $q=(x x 0)$ Зоны Бриллюэна, где дважды вырожденная мода расщепляется на продольную и поперечную. Собственные вектора оптической моды представляют собой линейную комбинацию смещений $\mathrm{PbZr}(\mathrm{Ti})$ и $\mathrm{O}_{3}$ в плоскости $X-Y$. Относительные вклады этих смещений определяются деталями межатомных взаимодействий. К настоящему времени отсутствуют теоретические расчеты, описывающие динамику решетки при конечных температурах. В связи с этим, при обработке $F_{\mathrm{O}}^{2}$ считался независимым параметром. Частоты и константы затухания акустической и оптической мод полагались не зависящими от температуры.

Для аппроксимации данных формулой (8) использовался метод переменной метрики MIGRAD [30] из пакета MINUIT C++ через интерфейс iminuit Python. Результаты подгонки показаны линиями на рис. 1. На рис. 1, $a$ приведен спектр, измеренный при $700 \mathrm{~K}$ в точке $Q=\left(\begin{array}{lll}2.5 & 2.50\end{array}\right)$, где зануляется. Все остальные спектры (рис. $1, b, c, d, f)$ измерены при $Q=\left(\begin{array}{lll}3.5 & 0.5 & 0\end{array}\right)$. Видно, что при $700 \mathrm{~K}$ вклад АФД-моды очень невелик, и мог быть обнаружен только благодаря одновременной обработке спектров в двух точках и фиксации величин неупругих структурных факторов, рассчитанных с использованием приведенных выше собственных векторов. При понижении температуры и приближении к точке фазового перехода происходит понижение частоты АФД-моды и пики, связанные с рождением и уничтожением фононов сливаются. Начиная с $625 \mathrm{~K}$ происходит переход в переторможенный режим (overdamped), при этом наблюдается один пик при $\omega=0$, ширина которого определяется отношением $\omega_{\mathrm{AFD}}^{2} / \Gamma_{\mathrm{AFD}}^{2}$.

На рис. 2 приведена температурная зависимость квадрата частоты АФД моды. Видно, что она хорошо описывается законом Кюри-Вейса $\omega_{\mathrm{AFD}}^{2} \propto\left(T-T_{\mathrm{AFD}}\right)$ с $T_{\mathrm{AFD}}=438 \pm 5 \mathrm{~K}$. Можно обратить внимание на тот факт, что в близком по составу соединении $\mathrm{PbZr}_{0.99} \mathrm{Ti}_{0.01} \mathrm{O}_{3}$ критическая температура, опре- 
деленная по данным диэлектрических измерений, $T_{\mathrm{FE}}=479.5 \mathrm{~K}[6]$ лишь ненамного выше, чем $T_{\mathrm{AFD}}$. Такая близость заставляет поднять вопрос о взаимодействии АФД моды и мягкой моды в центре зоны Бриллюэна, ответственной за СЭ фазовый переход. Очевидно, что линейного члена, описывающего взаимодействие существовать не может, однако биквадратичный всегда разрешен. Следует отметить еще один важный момент. В М-точке частота кислородной АФД-моды оказывается ниже частоты акустических колебаний. Учитывая, что при отклонении от этой точки (по крайней мере в направлениях отличных от линии, соединяющей Ми R-точки) частота кислородной моды быстро растет, неизбежным оказывается возникновение межмодового взаимодействия, приводящего к „антикроссингу“ дисперсионных кривых. Можно предположить, что именно такое взаимодействие ответственно за тот факт, что законы погасания для обнаруженной нами мягкой моды и сверхструктурных отражений в районе М-точки не совпадают (сверхструктурные отражения $(h+1 / 2 k+1 / 2 l)$ наблюдаются при всех $h, k, l$, в то время как мягкая $M_{3}$-мода проявляется только при $h \neq k$ ).

Необходимо сделать еще одно замечание. Учитывая приведенные результаты, следует отметить неоднозначность отнесения обнаруженного в морфотропном PZT мягкого возбуждения к моде $M_{5^{\prime}}$ [23]. Проведенные нами непосредственные расчеты неупругих структурных факторов подтверждают наблюдаемость АФД кислородной моды и, соответственно, необходимость рассмотрения ее вклада в наблюдаемые спектры.

\section{6. Заключение}

Проведенные нами измерения неупругого рассеяния рентгеновского (синхротронного) излучения позволили выявить существование в кристалле PZT2.4 мягкого возбуждения в точке М зоны Бриллюэна. Анализ правил отбора и прямой расчет неупругих структурных факторов позволили однозначно соотнести обнаруженное мягкое возбуждение с антиферродисторсионной кислородной моды с симметрией $M_{3}$. Частота этой моды подчиняется закону Кюри-Вейсса с критической температурой $T_{\mathrm{AFD}}=438 \pm 5 \mathrm{~K}$, близкой к сегнетоэлектрической температуре Кюри $T_{\mathrm{FE}}=479.5 \mathrm{~K}$, что поднимает вопрос о возможном взаимодействии сегнетоэлектрической и антиферродисторсионной мод. Частота АФД-моды в М-точке оказывается ниже частоты акустической моды в этой точке. Такое соотношение частот приводит к неизбежности возникновения при отклонении от М-точки (в частности, в направлении [110]) межмодового взаимодействия и „антикроссинга““.

\section{Благодарности}

С. Б. Вахрушев благодарит РФФИ за финансовую поддержку, грант № 20-02-00724-а

\section{Конфликт интересов}

Авторы заявляют, что у них нет конфликта интересов.

\section{Список литературы}

[1] Z. Ujma, J. Hańderek. Phys. Status Solidi A 28, 2, 489 (1975).

[2] R.W. Whatmore, A.M. Glazer. J. Physics C 12, 8, 1505 (1979).

[3] K. Roleder, J. Dee. J. Physics: Condens. Matter 1, 8, 1503 (1989).

[4] J.-H. Ko, M. Górny, A. Majchrowski, K. Roleder, A. Bussmann-Holder. Phys. Rev. B 87, 18, (2013).

[5] R. Faye, H. Liu, J.M. Kiat, B. Dkhil, P.E. Janolin. Appl. Phys. Lett. 105, 16, 162909 (2014).

[6] K. Roleder, I. Jankowska-Sumara, G.E. Kugel, M. Maglione, M.D. Fontana, J. Dec. Phase Transitions 71, 4, 287 (2006).

[7] R.G. Burkovsky, I. Bronwald, D. Andronikova, B. Wehinger, M. Krisch, J. Jacobs, D. Gambetti, K. Roleder, A. Majchrowski, A.V. Filimonov, A.I. Rudskoy, S.B. Vakhrushev, A.K. Tagantsev. Sci. Rep. 7, (2017).

[8] A. Bosak, V. Svitlyk, A. Arakcheeva, R. Burkovsky, V. Diadkin, K. Roleder, D. Chernyshov. Acta Crystallogr. B 76, 1, 7 (2020).

[9] D. Viehland, J.-F. Li, X. Dai, Z. Xu. J. Phys. Chem. Solids 57, 10, 1545 (1996).

[10] J. Ricote, D.L. Corker, R.W. Whatmore, S.A. Impey, A.M. Glazer, J. Dec, K. Roleder. J. Physics: Condens. Matter 10, 8, 1767 (1998).

[11] D.I. Woodward, J. Knudsen, I.M. Reaney. Phys. Rev. B 72, 10, (2005).

[12] Z. An, H. Yokota, N. Zhang, M. Paściak, J. Fábry, M. Kopecký, J. Kub, G. Zhang, A.M. Glazer, T.R. Welberry, W. Ren, Z.-G. Ye. Phys. Rev. B 103, 5, (2021.)

[13] D. Viehland. Phys. Rev. B Condens. Matter 52, 2, 778 (1995).

[14] D.A. Andronikova, I.A. Bronwald, N.G. Leontyev, I.N. Leontyev, D.Y. Chernyshov, A.V. Filimonov, S.B. Vakhrushev. Phys. Solid State 61, 10, 1772 (2019).

[15] S. Watanabe, Y. Koyama. Phys. Rev. B 66, 13, (2002).

[16] T. Ostapchuk, J. Petzelt, V. Zelezny, S. Kamba, V. Bovtun, V. Porokhonskyy, A. Pashkin, P. Kuzel, M. Glinchuk, I. Bykov. J. Physics: Condens. Matter 13, 11, 2677 (2001).

[17] A.K. Tagantsev, K. Vaideeswaran, S.B. Vakhrushev, A.V. Filimonov, R.G. Burkovsky, A. Shaganov, D. Andronikova, A.I. Rudskoy, A.Q. Baron, H. Uchiyama, D. Chernyshov, A. Bosak, Z. Ujma, K. Roleder, A. Majchrowski, J.H. Ko, N. Setter. Nature Commun. 4, 1, (2013).

[18] R.G. Burkovsky, A.K. Tagantsev, K. Vaideeswaran, N. Setter, S.B. Vakhrushev, A.V. Filimonov, A. Shaganov, D. Andronikova, A.I. Rudskoy, A.Q.R. Baron, H. Uchiyama, D. Chernyshov, Z. Ujma, K. Roleder, A. Majchrowski, J.-H. Ko. Phys. Rev. B 90, 14, (2014).

[19] R.G. Burkovsky, I. Bronwald, D. Andronikova, G. Lityagin, J. Piecha, S.-M. Souliou, A. Majchrowski, A. Filimonov, A. Rudskoy, K. Roleder, A. Bosak, A. Tagantsev. Phys. Rev. B 100, 1, (2019).

[20] M. Paściak, T.R. Welberry, A.P. Heerdegen, V. Laguta, T. Ostapchuk, S. Leoni, J. Hlinka. Phase Transitions 88, 3, 273 (2014). 
[21] N. Zhang, M. Paściak, A.M. Glazer, J. Hlinka, M. Gutmann, H.A. Sparkes, T.R. Welberry, A. Majchrowski, K. Roleder, Y. Xie, Z.-G. Ye. J. Appl. Crystallography 48, 6, 1637 (2015).

[22] D.A. Andronikova, I.A. Bronwald, I.N. Leontiev, N.G. Leontiev, D.Y. Chernyshov, A.V. Filimonov, S.B. Vakhrushev. Ferroelectrics 538, 1, 65 (2019).

[23] J. Hlinka, P. Ondrejkovic, M. Kempa, E. Borissenko, M. Krisch, X. Long, Z.G. Ye. Phys. Rev. B 83, 14, (2011).

[24] P. Ghosez, E. Cockayne, U.V. Waghmare, K.M. Rabe. Phys. Rev. B 60, 2, 836 (1999).

[25] D.A. Andronikova, I.A. Bronwald, R.G. Burkovsky, I.N. Leontiev, N.G. Leontiev, A.A. Bosak, A.V. Filimonov, S.B. Vakhrushev. J. Physics: Conf. Ser. 769, 012070 (2016).

[26] Н. Леонтьев, В.Г. Смотраков, Е.Г. Фесенко. Изв. АН СССР. Неорган. материалы 18, 3, 449 (1982).

[27] П.П. Сырников. ФТТ 25, 8, 2529 (1983).

[28] R. Cowley. Phys. Rev. 134, 4A, (1964).

[29] Ю. Изюмов, Н. Черноплеков. Нейтронная спектроскопия. Энергоатомиздат, М. (1983) $328 \mathrm{c.}$

[30] F. James, M. Roos. Comput. Phys. Commun. 10, CERN-DD-75-20, 343 (1975).

Редактор Т.Н. Василевская 